\title{
An Adaptive Genetic Algorithm approach for predicting magnetic structure suitable for high- performance permanent magnet development
}

\author{
P. Nieves, S. Arapan and S. Cuesta-López \\ ICCRAM, International Research Center in Critical Raw Materials and Advanced Industrial \\ Technologies, University of Burgos, 09001 Burgos, Spain
}

The uncertainty in rare-earth (RE) market resulted in worldwide efforts to develop RElean/free permanent magnets (PMs). In order to solve this problem many efforts are focused on reducing and recycling the content of RE elements in PM. An alternative strategy to solve the RE problem in PM is to search for new high-performance RE-free PM. So far the historical development of PM shows that experimental approaches were the most successful way for finding new and better PM. However, this traditional trend may reverse due to theoretical advances in materials science and powerful computational tools that are available at the present time, which could be essential in the discovery of the so desired next-generation of PM. One of the most important and difficult step in the high-throughput computational technique for materials design is the prediction of new stable crystal phases. So far, many methods have been developed for predicting crystal structure as the simulated annealing, basin hopping, ab-initio random structure search, metadynamics, particle swarm optimization, cluster expansion method and adaptive genetic algorithm (AGA) [1].

AGA are adaptive heuristic algorithms used to solve optimization problems based on evolutionary ideas of natural selection and genetics. AGA belongs to a larger class of evolutionary algorithms that generates solutions to optimization problems using various evolutionary techniques, such as inheritance, selection, mutation and crossover. Evolution is considered as an iterative process, which starts with a random generation of individuals of a population (a generation). Individuals are candidate solutions to the optimization problem and within AGA the population is evolved toward better solutions. In the case of the structure predicting an individual is a randomly generated crystal structure (cell lattice parameters and ion position) and the solution is the ground state phase of the system at certain pressure (P) and temperature $(T)$ conditions (the structure with the lowest enthalpy for $T=0 \mathrm{~K}$ ).

In this work, we present a general overview, analysis and software implementation of a particular AGA, which has been proposed for discovering new RE-free magnetic crystal phases in the context of the EU-H2020 NOVAMAG project. Fig.1 shows the workflow diagram related to the Modelling Data Elements (MODA) of the magnetic crystal structure calculation based on AGA. In particular, we make use of an AGA, implemented through USPEX [2] and VASP [3] codes, to predict new magnetic crystal phases, where those structures with better magnetic properties for a permanent magnet are selected and analyzed in more detail. Such a methodology has been preliminary compared to well-known experimentally reported properties compounds, showing an excellent agreement. In addition, we show recent results predicted by $A G A$ in $\mathrm{CoFe}_{2 n} \mathrm{X}(\mathrm{n}=1,2,3,4,5$; where $\mathrm{X}=\mathrm{C}, \mathrm{P}, \mathrm{Hf}, \mathrm{Zr}, \ldots)$, where we found compounds with many metastable structures which fulfill the initial requirements (negative enthalpy of formation $\Delta H_{F}<0$, high saturation magnetization $\mu_{0} M_{s}>1 T$ and non-cubic lattice system) for permanent magnet development, see Fig.2. 


\section{ACKNOWLEDGEMENT}

Authors acknowledge support from NOVAMAG project, under Grant Agreement No. 686056, EU Horizon 2020 Framework Programme for Research and Innovation (2014-2020). Authors also acknowledge the Spanish Supercomputing Network (RES) and CESVIMA for providing supercomputational resources under Ref: QCM-2016-2-0034.

\section{REFERENCES}

[1] M. Ji, K. Umemoto, C. Z. Wang, K. M. Ho and R. M. Wentzcovitch, Phys. Rev. B 84, 220105 (2011).

[2] A. O. Lyakhov, A. R. Oganov, H. T. Stokes, Qi-ang Zhu, Computer Physics Communications 184, 1172-1182 (2013).

[3] G. Kresse and J. Hafner, Phys. Rev. B 47, 558 (1993); ibid. 49, 14251 (1994).

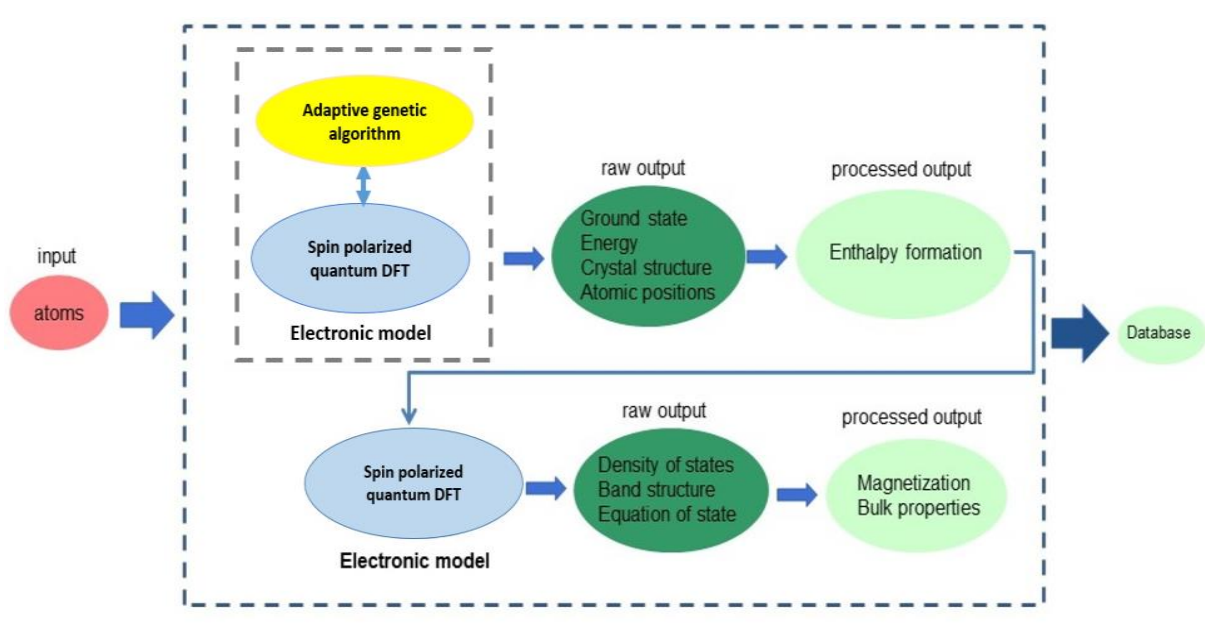

Figure 1. Workflow diagram related to MODA of the magnetic crystal structure calculation.
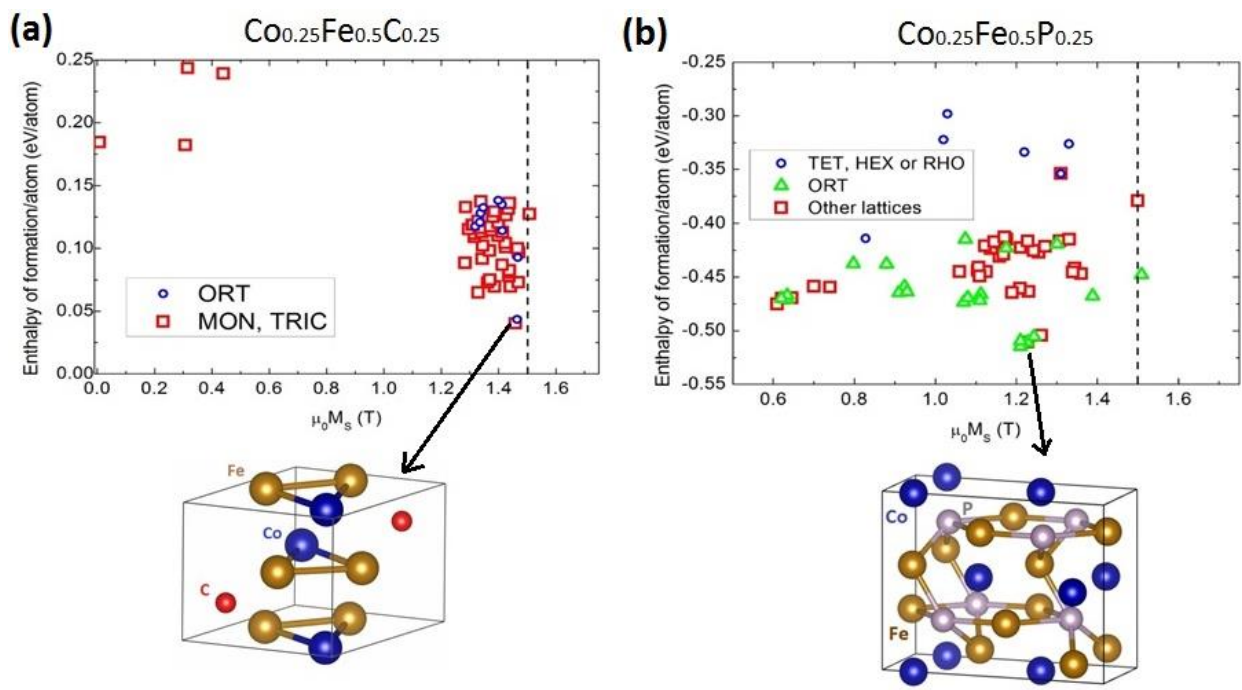

Figure 2. Estimated enthalpy of formation versus saturation magnetization of the structures found by AGA for the compounds: (a) $\mathrm{Co}_{0.25} \mathrm{Fe}_{0.5} \mathrm{C}_{0.25}$ and (b) $\mathrm{Co}_{0.25} \mathrm{Fe}_{0.5} \mathrm{P}_{0.25}$. 